\title{
Optical, Scintillation and Dosimeter Properties of MgO Transparent Ceramic and Single Crystal
}

\author{
Takumi Kato, Go Okada and Takayuki Yanagida \\ Nara Institute of Science and Technology (NAIST) \\ 8916-5, Takayama-cho, ikoma-shi, Nara 630-0192, Japan \\ Phone:+81-743-72-6144 Email: kato.takumi.ki5@ms.nasit.jp
}

\begin{abstract}
We have synthesized a MgO transparent ceramic by spark plasma sintering (SPS) and studied the dosimeter properties for $\mathrm{X}$-ray detections, in comparison with the single crystal form. Further, we have characterized the optical and scintillation properties. Despite the same material compositions, the photoluminescence (PL) of the ceramic sample showed dominantly around 450 nm whereas the single crystal sample had a dominant emission in the NIR range. The PL lifetime of $450 \mathrm{~nm}$ were measured to be $\sim 10$ and $\sim 50 \mathrm{~ns}$ which were typical for emissions by $\mathrm{F}^{+}$center in $\mathrm{MgO}$. The scintillation spectra were similar to those observed in PL, but the lifetime was much longer on the micro-second scale. The TSL glow curves of $\mathrm{MgO}$ ceramic showed a main peak around $140{ }^{\circ} \mathrm{C}$. The TSL spectrum at $140{ }^{\circ} \mathrm{C}$ had a broad emission band in the 300-400 range and around 600 and $750 \mathrm{~nm}$, which approximately coincide with the scintillation spectra. The TSL response was confirmed to be linear to the irradiation dose for both samples over the dose range from 0.1 to 1000 mGy.
\end{abstract}

Keywords: transparent ceramic, MgO, scintillator, dosimeter, TSL 


\section{Introduction}

The accurate determination of the dose, energy and position of ionizing radiation has attracted much attention in the medical applications [1], security [2] and also for personal dose monitorings [3-5]. Specific properties required for a high precision dosimeter are a suitable sensitivity, dose linearity, energy response and low fading. In addition, it is desirable that the effective atomic number of the dosimeter material in the view point of bioequivalence should be close to that of human body soft tissue $\left(\mathrm{Z}_{\text {eff }}=7.13\right)$. If the effective atomic number of the dosimeter is the same as that of tissue, no mathematical dose reconstruction for energy dependence are required. Therefore, for dosimeter applications, materials consisting of light elements are preferable. Dosimeters are mainly classified to three types with respect to the mechanisms involved: thermally stimulated luminescence (TSL), optically stimulated luminescence (OSL) and radiophotoluminescence (RPL). Examples of these dosimeter materials used in practice are: Ti and $\mathrm{Mg}$ doped $\mathrm{LiF}$ ceramics [3], $\mathrm{C}: \mathrm{Al}_{2} \mathrm{O}_{3}$ crystalline powder [4] and Ag-doped phosphate glass [5], respectively.

Magnesium oxide $(\mathrm{MgO})$ is a wide band-gap insulator $(7.8 \mathrm{eV})$ with the rock-salt crystal structure (fcc) at ambient pressure, and the Mg ions occupy the octahedral sites within the anion closed packed structure $[6,7]$. The luminescence properties of undoped $\mathrm{MgO}$ have been studied in many material forms including powders, films, bulk single crystals and ceramics [8-17]. In MgO crystals, it has been demonstrated that there exist two primary oxygen vacancies, namely $\mathrm{F}^{+}$and $\mathrm{F}$ color centers, which capture one and two electrons, respectively. Although absorption bands of the $\mathrm{F}^{+}$and $\mathrm{F}$ color centers are almost the same energy of $\sim 5 \mathrm{eV}(\sim 250 \mathrm{~nm})$, they show different photoluminescence (PL) bands with the peaks at $\sim 2.3 \mathrm{eV}$ $(\sim 500 \mathrm{~nm})$ and $\sim 3.2 \mathrm{eV}(\sim 400 \mathrm{~nm})$, respectively, [13].

In the 1970s, undoped $\mathrm{MgO}$ powder was found to show dosimeter properties. When it was irradiated by X-rays, $\gamma$-rays, and UV lights, two TSL peaks were found around 90-100 and $140{ }^{\circ} \mathrm{C}$ [18-20]. Subsequently, in the later decades, $\mathrm{MgO}$ have been intensively studied for dosimeter applications [21-25] due to the bioequivalency. In recent years, with advancement of ceramic fabrication techniques, $\mathrm{MgO}$ transparent ceramic was developed [26-30], which shows long afterglow (or persistent luminescence) emission at the wavelength of $390 \mathrm{~nm}$ due to the $\mathrm{F}^{+}$center [30]. The long afterglow, in other words, is a form of TSL at room temperature; therefore, the $\mathrm{MgO}$ transparent ceramic has an ability to store radiation- (or photo-) induced charges and is potentially applicable for TSL dosimeter.

In this study, we have synthesized a MgO transparent ceramic by spark plasma sintering (SPS) and studied the dosimeter properties against X-rays, in comparison with a $\mathrm{MgO}$ single crystal. Generally, synthesis of SPS is done in highly reductive atmosphere; therefore, oxygen vacancies are effectively created and the dosimeter properties are expected to be enhanced. Further, we have report also the optical and scintillation properties because it is pointed out that scintillation and storage luminescence by ionizing radiation are complementarily related in recent study $[31,32]$. 


\section{Experiment}

A MgO transparent ceramic sample was synthesized by the SPS method using a Sinter Land LabX-100. Here, a reagent grade of $\mathrm{MgO}$ powder $\left(99.99 \%\right.$ ) was loaded in a graphite die and sintered at $1500{ }^{\circ} \mathrm{C}$ for $60 \mathrm{~min}$ while applying the pressure of $80 \mathrm{MPa}$. After the synthesis, wide surfaces of the ceramic sample were polished. The $\mathrm{MgO}$ single crystal sample used in this study is a commercial product and was purchased from NEOTRON, Japan. As same for the ceramic sample, the wide surfaces were polished. In the course of study, the following measurements were carried out for these two samples.

The in-line transmittance was evaluated by using a JASCO V670 spectrometer in the spectral range from 190 to $2700 \mathrm{~nm}$ with $1 \mathrm{~nm}$ step [33]. The photoluminescence (PL) emission spectrum was measured with a $265 \mathrm{~nm}$ excitation source produced by a combination of xenon lamp (LAX-C100, Asahi Spectra) and a band-pass filter (VPF-25C-10-12-26500, OptoSigma). The consequent emission spectrum was digitized using an optical fiber-coupled CCD spectrometer (QE65Pro, Ocean Optics). PL decay lifetime monitoring at $410 \mathrm{~nm}$ was measured with $280 \mathrm{~nm}$ excitation using Hamamatsu Quantaurus- $\tau$ (C11367-04, Hamamatsu). The X-ray induced scintillation spectrum was measured using our lab-constructed setup. The sample was excited using the X-ray generator in which the applied tube voltage and current were 40 $\mathrm{kV}$ and $5.2 \mathrm{~mA}$, respectively. The scintillation emission was guided to either of the following two CCDbased spectrometers: Andor (CCD, DU920-BU2NC; grating, SR163i-UV) and Ocean Optics (QE65Pro). The former was used for measuring a spectrum in the UV and visible range while the latter was used for the NIR range. Further, the scintillation lifetime by X-ray irradiation was measured using an afterglow characterization system equipped with a pulsed X-ray tube [34]. The system is commercially available from Hamamatsu as a custom-ordered instrument. The applied voltage to the pulsed X-ray source was 30 $\mathrm{kV}$, and the system offers the timing resolution of $\sim 1 \mathrm{~ns}$.

In order to investigate the properties of $\mathrm{MgO}$ ceramic as a thermally-stimulated luminescent (TSL) dosimetric detector, we have measured a TSL glow curve using a Nanogray TL-2000 [35] after varying X-ray irradiation doses from $0.1 \mathrm{mGy}$ to $1000 \mathrm{mGy}$. The heating rate used for all the TSL measurements was fixed to $1{ }^{\circ} \mathrm{C} / \mathrm{s}$ over the temperature range from 50 to $490{ }^{\circ} \mathrm{C}$. The TSL spectrum was measured using Andor spectrometer (CCD, DU920-BU2NC; grating, SR163i-UV) while the sample was heated by an electric heater (SCR-SHQ-A, Sakaguchi E.H Voc) at a constant temperature.

\section{Results and discussion}

\section{1 Sample}

The $\mathrm{MgO}$ ceramic sample synthesized by the SPS method was visibly transparent. Fig.1 (top) shows a photograph of the $\mathrm{MgO}$ ceramic and single crystal samples. The bottom figure in Fig. 1 showed the samples under UV (254 nm) excitation, which are emitting light of yellow-green color. Fig. 2 illustrates an SEM image of the MgO ceramic sample. From the SEM image, it can be confirmed that the sample was properly creamed. The grain size and shape seem to be non-uniform. However, the adjacent grains 
are closely attached; therefore, a scattering of light is minimized and it appears to be transparent as also confirmed in Fig. 1 (top).

\section{2 Optical properties}

Fig. 3 shows the in-line transmittance spectrum of $\mathrm{MgO}$. The $\mathrm{MgO}$ ceramic and single crystal showed a transmittance around $20-70 \%$ and $50-80 \%$ at wavelengths longer than $300 \mathrm{~nm}$, respectively. At $190 \mathrm{~nm}$ and shorter wavelengths, the transmittance is close to zero. This range is, in fact, longer than the wavelength that corresponds to the bandgap energy of $\mathrm{MgO}(\sim 7.8 \mathrm{eV}=159 \mathrm{~nm})$ [6]. Therefore, we think that this is due to insufficient sensitivity of the instrument in this spectral range.

Fig. 4 represents the PL spectrum of the $\mathrm{MgO}$ ceramic and single crystal samples under $265 \mathrm{~nm}$ excitation. Main emission band of both samples appeared at $410 \mathrm{~nm}$, but the emission intensities were largely different despite the fact that the sample sizes are comparable. Moreover, in the ceramic sample, a single small emission was observed at $700 \mathrm{~nm}$ (inset of Fig. 4 right). We think that the $700 \mathrm{~nm}$ emission band is due to $\mathrm{Cr}$ ion because it was previously reported that a broad emission arises in the spectral range around $700 \mathrm{~nm}$ by $\mathrm{Cr}$ ion [19], which can typically contaminate in $\mathrm{MgO}$. Furthermore, strong emissions of a broad band with sharp lines appeared around near infrared (NIR) wavelength of 750-950 $\mathrm{nm}$ in the crystal sample.

PL decay time profiles monitoring at $410 \mathrm{~nm}$ are shown in Fig. 5. The decay curves are well approximated by a three exponential function. In the $\mathrm{MgO}$ single crystal, the obtained decay time constants are 1.9, 5.6 and $18 \mathrm{~ns}$. In the ceramic sample, decay time constants of 2.1, 7.2 and 47.1 ns were observed. We tentatively attribute the origin of each component as follows. The fastest components (1.9 and $2.1 \mathrm{~ns}$ ) of both samples would be due to the instrumental response because the timing resolution of the instrument is comparable (around $1 \mathrm{~ns}$ ). Although we deconvoluted the instrumental response, some residual signals were still present. The second fastest (5.6 and $7.2 \mathrm{~ns}$ ) and slow (18 and $47 \mathrm{~ns})$ decay time components of both samples are in the typical range for emissions from $\mathrm{F}^{+}$center [8-17]. Therefore, the peak at $410 \mathrm{~nm}$ was assigned to the emission due to $\mathrm{F}^{+}$center. PL decay time profile of crystal sample monitoring $750 \mathrm{~nm}$ is shown in Fig. 6. The decay curve showed two decay time components: one was fast $0.3 \mathrm{~ms}$ and the other was slow $4.4 \mathrm{~ms}$. Since the decay time scale is considerably long, we suspect that these sharp emission lines of 750-950 $\mathrm{nm}$ are due to an unexpected contamination such as rare earth ions included in the synthesis processes because many rare earth ions tend to show such sharp emission lines regardless of the host crystal fields by the inter $4 \mathrm{f}$ shell transitions.

\section{3 Scintillation properties}

Fig. 7 shows the scintillation spectra of the $\mathrm{MgO}$ ceramic and single crystal samples. Since both samples do not intentionally include any luminescent centers, the origins of these emissions must be other types of luminescence mechanisms such as exciton and defect centers, or unexpected impurities. The 
emission bands of the $\mathrm{MgO}$ ceramic had broad spectral shapes with the peaks at 340, 600 and $760 \mathrm{~nm}$. These peak locations are consistent with the ones seen in the PL spectrum under $265 \mathrm{~nm}$ excitation. The $340 \mathrm{~nm}$ peak is reasonably consistent with the earlier work [13], and the origin of this emission was attributed to the $\mathrm{F}^{+}$center. The origin of $760 \mathrm{~nm}$ peak was attributed to the $\mathrm{Cr}$ ion, as similarly seen in the PL spectrum. The origin of emission band at $600 \mathrm{~nm}$ is currently under investigation. On the other hand, the $\mathrm{MgO}$ single crystal showed some scintillation emissions with the peaks at $600 \mathrm{~nm}$ and 850-900 $\mathrm{nm}$. Unlike PL, no broad emission was observed around $340 \mathrm{~nm}$. The origin of emission of $600 \mathrm{~nm}$ is currently under investigation. In addition, the origins of sharp emissions in the 850-950 nm is ascribed to the unexpected rare earth contamination by the same reasons for the PL discussed above. For the emissions by $\mathrm{F}^{+}$center at $340 \mathrm{~nm}$, it is much more pronounced in the ceramic sample than single crystal. The reason can be explained that, since the sample was synthesized in a high reducing atmosphere, the ceramic sample contains more oxygen vacancies, each of which attract a single hole charge to form a $\mathrm{F}^{+}$ center.

In Fig. 8, the X-ray exited scintillation decay time profiles are shown. As for the PL decay time profiles, the scintillation decay curves are well approximated by a three exponential function. The deducted decay time constants are $0.007,0.4$ and $2.3 \mu$ s for the ceramic, and $0.004,0.06$ and $37 \mu$ s for the single crystal sample. The fastest component is from the instrument since the response time of the PMT (R7400, Hamamatsu) is around $1 \mathrm{~ns}$. The decay lifetimes are much slower than those by PL. This difference arises by the different way of excitation and different emission mechanisms. While the X-ray ionize the host matrix and then the generated charges recombine at the luminescent centers to emit light, the UV excitation directly excites the electronic state of luminescent center followed by the emission. Since it tend to take longer time for charge/energy transfer to the luminescent center in the scintillation process, it is typical to have a longer lifetime in scintillation than PL.

\section{4 Dosimeter properties}

Fig. 9 shows TSL glow curves of the ceramic and single crystal samples measured after the samples were irradiated at $1 \mathrm{~Gy}$. Both the samples showed a strong single glow peak around $140{ }^{\circ} \mathrm{C}$. This peak is consistent with previous works reported in literatures [18-24], which is a typical feature of $\mathrm{MgO}$. Further, there are some additional glow peaks observed around 240 and $350^{\circ} \mathrm{C}$ for the ceramic sample and 320 , $400^{\circ} \mathrm{C}$ for the single crystal sample. This fact indicates that there exist three different trapping centers in both ceramic and single crystal samples. Particularly, the peak at $\sim 400^{\circ} \mathrm{C}$ in the crystal is related to the thermal decay of $\mathrm{H}$ centers as discussed in [23], in which the interstitial anion recombines with a neighboring $\mathrm{F}^{+}$center followed by emitting light. Thus, in the ceramic produced by SPS, it is suggested that there exists less anion interstitials, and the latter was also suggested by the scintillation spectrum where the emission by $\mathrm{F}^{+}$center is larger for the ceramic sample. Overall, TSL intensity is, in fact, much stronger for the ceramic sample than crystal while, as shown in Fig. 6, the scintillation intensity is much 
stronger for the crystal. As discussed by Yanagida et al. [31, 32], these two emission intensities by different radiation-induced emission mechanisms, that is, as a dosimeter or scintillator, are typically in a complemental relationship. In a bright scintillator material, the most of radiation generated charges transfer to the luminescent center to emit light while there are less charges trapped, and vice versa.

Fig. 10 depicts a TSL spectrum of the $\mathrm{MgO}$ ceramic around $140{ }^{\circ} \mathrm{C}$. The spectrum had a broad emission band in 300-400, 600 and $750 \mathrm{~nm}$, which approximately coincide with scintillation spectra. However, the emission intensity at $750 \mathrm{~nm}$ is the strongest unlike in the case of the PL and scintillation spectra. This indicates that the $\mathrm{Cr}$, even with a contamination content, acts as a very effective luminescent center in TSL. In addition, it should be noted that the intense $750 \mathrm{~nm}$ emission is not included in the glow curve (Fig. 9) since the thermal radiation cut filter was equipped in the instrument. If this emission signal were included, a much lower dose would be detected. Furthermore, TSL spectrum of MgO single crystal could not be measured for its weak intensity.

Fig. 11 shows a relationship between the TSL intensity and irradiated X-ray dose, namely the dose response curve, of the $\mathrm{MgO}$ transparent ceramic and single crystal samples. The dose response is an important characteristic as dosimeter applications. The TSL signal used here is the integrated signal of the peak at $140{ }^{\circ} \mathrm{C}$ shown in Fig. 9. From our experimental results, both the samples have a linear response to the incident X-ray dose over the dose range from 0.1 to $1000 \mathrm{mGy}$. Dosimeter having a linear response is advantageous because it enables us to easily and accurately calibrate the dose. Furthermore, particularly the $\mathrm{MgO}$ ceramic has much higher sensitivity than that of the single crystal. It is an equivalent performance with commercial dosimeters for personal dosimetry and showed much more superior performance for $\mathrm{MgO}$ than ever reported.

\section{Conclusions}

We have synthesized $\mathrm{MgO}$ transparent ceramics by an SPS technique. Subsequently, optical, scintillation and dosimeter properties were investigated in comparison with a $\mathrm{MgO}$ single crystal. The TSL glow curves of both samples have the main peak around $140{ }^{\circ} \mathrm{C}$. Using this glow peak, both the samples have a dose detection ability at least over the range 0.1-1000 mGy. Furthermore, the response is linearly correlated with the incident dose over the dose range tested.

\section{Acknowledgements}

This work was supported by a Grant in Aid for Scientific Research (A)-26249147 from the Ministry of Education, Culture, Sports, Science and Technology of the Japanese government (MEXT) and partially by JST A-step. Green Photonics Research from MEXT and the Cooperative Research Project of Research Institute of Electronics, Shizuoka University, are also acknowledged.

\section{References}

[1] T. Yanagida, A. Yoshikawa, Y. Yokota, K. Kamada, Y. Usuki, S. Yamamoto, M. Miyake, M. Baba, 
K. Sasaki and M. Ito, IEEE Trans. Nucl. Sci., 571492 (2010).

[2] D. Totsuka, T. Yanagida, K. Fukuda, N. Kawaguchi, Y. Fujimoto, Y. Yokota and A. Yoshikawa, Nucl. Instrum. Methods A., 659399 (2011).

[3] M. R. Mayhugh, R. W. Chrisy and N. M. Johnson, J. Appl. Phys., 412968 (1970).

[4] S.W.S. McKeever, Radiat. Meas., 461336 (2011).

[5] Y. Miyamoto, Y. Takei, H. Nanto, T. Kurobori, A. Konnai, T. Yanagida, A. Yoshikawa, Y. Shimotsuma, M. Sakakura, K. Miura, K. Hirao, Y. Nagashima and T. Yamamoto, Radiat. Meas., 46 1480 (2011).

[6] D.M. Roessler, W.C. Walker, Phys. Rev., 159733 (1967).

[7] C. Klein, C.S. Hurlbut Jr., Manual of Mineralogy, John Wiley \& Sons, New York, 1999.

[8] T. Uchino, D. Okutsu, R. Katayama, S. Sawai, Phys. Rev. B., 79165107 (2009)

[9] H. Soma, Y. Uenaka, A. Asahara, T. Suemoto, T. Uchino, Appl. Phys. Lett., 106041116 (2015).

[10] Y. Yanagisawa, R. Huzimura, J. Phys. Soc. Jpn., 5366 (1984).

[11] G.P. Summers, T.M. Wilson, B.T. Jeffries, H.T. Tohver, Y. Chen, M.M. Abraham, Phys. Rev. B., 27 1283 (1983).

[12] C. Martinez-Boubeta, A. Martinez, S. Hernandez, P. Pellegrino, A. Antony, J. Bertomeu, Ll. Balcells, Z. Konstantinovic, B. Martinez, Solid. State. Commun., 151751 (2011).

[13] G.H. Rosenblatt, M.W. Rowe, G.P. Williams Jr., R.T. Williams, Y. Chen, Phys. Rev. B., 3910309 (1989).

[14] Y. Chen, J.L. Kolopus, W.A. Sibley, Phys. Rev. B., 186865 (1969).

[15] L.A. Kappers, R.L. Kroes, E.B. Hensley, Phys. Rev. B., 14151 (1970).

[16] T. Jeffries, R. Gonzalez, Y. Chen, G.P. Summers, Phys. Rev. B., 252077 (1982).

[17] Y. Uenaka, T. Uchino, Phys. Rev. B., 83195108 (2011).

[18] H. Nanto, K. Inabe, H. Yamazaki, N. Takeuchi, J. Phys. Chem. Solids., 36477 (1975).

[19] N. Takeuchi, K. Inabe, H. Nanto, Solid. State. Commun., 171267 (1975).

[20] W.A. Sibley, J.L. Kolopus, W.C. Mallard, Phys. Status Solidi., 31223 (1969).

[21] W.M. Ziniker, J.M. Rusin, T.G. Stoebe, J. Mater. Sci., 8407 (1973).

[22] A. Sathyamoorthy, J.M. Luthra, J. Mater. Sci., 132637 (1978).

[23] W.C. Las, T.G. Stoebe, Radiat. Prot. Dosim., 845 (1984).

[24] S. Dolgov, T. Karner, A. Lushchik, A. Maaroos, N. Mironova-Ulmane, S. Nakonechnyi, Radiat. Prot. Dosim., 100127 (2002).

[25] C. Soliman, Radiat. Eff. Defect. S., 164257 (2009).

[26] Y. Fang, D. Agrawal, G. Skandan, M. Jain, Mater. Lett., 58551 (2004).

[27] R. Chaim, Z. Shen, M. Nygren, J. Mater. Res., 192527 (2004).

[28] D. Chen, E.H. Jordan, M. Gell, Scripta. Mater., 59757 (2008)

[29] R. Chaim, R. Marder, C. Estourns, Scripta. Mater., 63211 (2010).

[30] S. Wakahara, T. Yanagida, Y. Yokota, Y. Fujimoto, V. Chani, M. Sugiyama, Y. Futami, A. Yoshikawa, Opt. Mater., 35558 (2013).

[31] T. Yanagida, Y. Fujimoto, K. Watanabe, K. Fukuda, N. Kawaguchi, Y. Miyamoto, H. Nanto, Radiat. Meas., 71162 (2014)

[32] T. Yanagida, J. Lumin., in press, doi:10.1016/j.jlumin.2015.01.006 (2015).

[33] T. Yanagida, K. Kamada, Y. Fujimoto, H. Yagi, T. Yanagitani, Opt. Mater., 352480 (2013).

[34] T. Yanagida, Y. Fujimoto, T. Ito, K. Uchiyama, K. Mori, Appl. Phys. Exp., 7062401 (2014).

[35] T. Yanagida, Y. Fujimoto, N. Kawaguchi, S. Yanagida, J. Ceram. Soc. Jpn., 121988 (2013). 
Fig. 1 Synthesized MgO transparent ceramic (left) and single crystal sample (right) under ambient light (top) and $254 \mathrm{~nm}$ UV light (bottom).

Fig. 2 SEM image of $\mathrm{MgO}$ ceramic.

Fig. 3 Transmittance spectra of $\mathrm{MgO}$ transparent ceramic and single crystal.

Fig. 4 PL emission spectrum of MgO ceramic and single crystal samples when excited at $265 \mathrm{~nm}$ in the UV-Vis (left) and NIR ranges (right). The inset in the right figure expands the spectral data around 700 nm.

Fig. 5 PL decay time profiles of $\mathrm{MgO}$ ceramic (left) and single crystal (right) samples under $280 \mathrm{~nm}$ excitation while monitoring the $410 \mathrm{~nm}$ emission.

Fig. 6 PL decay time profiles of MgO single crystal sample under $280 \mathrm{~nm}$ excitation while monitoring the $750 \mathrm{~nm}$ emission.

Fig. $7 \mathrm{X}$-ray induced scintillation spectra of the $\mathrm{MgO}$ ceramic and the single crystal samples. The inset expands 650-950 $\mathrm{nm}$ regions.

Fig. 8 Scintillation decay time profiles of the $\mathrm{MgO}$ ceramic (left) and single crystal (right) samples.

Fig. 9 TSL glow curve of the MgO ceramic and the single crystal after 1 Gy X-ray exposure.

Fig. $10 \mathrm{TSL}$ spectra at $140^{\circ} \mathrm{C}$ glow peak of the $\mathrm{MgO}$ ceramic. The inset expands spectra from 250 to 700 $\mathrm{nm}$

Fig. 11 Dose response curve of the $\mathrm{MgO}$ ceramic and the single crystal. The TSL signal used here is the peak intensity observed at $140^{\circ} \mathrm{C}$. 
Fig. 1 Synthesized MgO transparent ceramic (left) and single crystal sample (right) under ambient light (top) and $254 \mathrm{~nm}$ UV light (bottom). 
1

2

3

4

5

6

7

8

10

11

12

13

14

15

16

17

18

19

20

21

22

23

24

25

26

27

28

29

30

31

32

33

34

35

36

37

38

39

40

41

42

43

44

45

46

47

48

49

50

51

52

53

54

55

56

57

58

59

60

61

62

63

64

65

Fig. 2 SEM image of $\mathrm{MgO}$ ceramic.

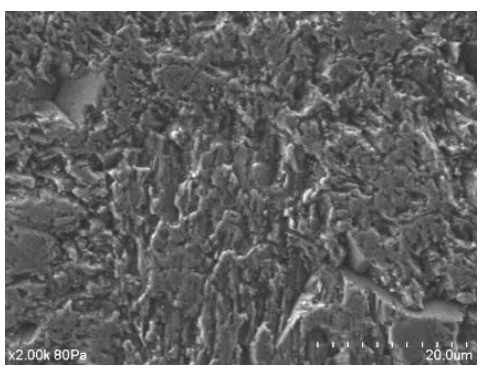


1

2

3

4

5

6

7

8

10

11

12

13

14

15

16

17

18

19

20

21

22

23

24

25

26

27

28

29

30

31

32

33

34

35

36

37

38

39

40

41

42

43

44

45

46

47

48

49

50

51

52

53

54

55

56

57

58

59

60

61

62

63

64

65

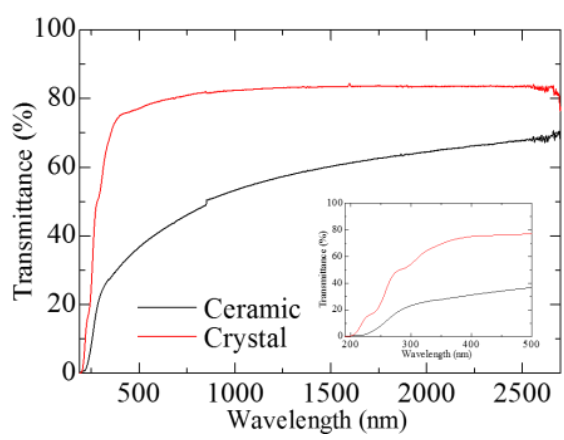

Fig. 3 Transmittance spectra of $\mathrm{MgO}$ transparent ceramic and single crystal. 
1

2
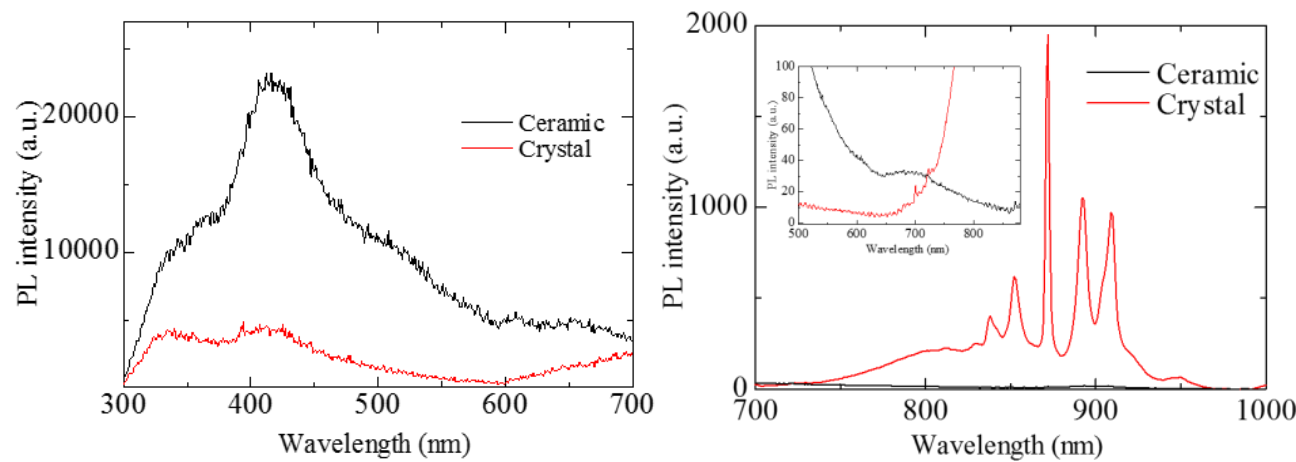

Fig. 4 PL emission spectrum of $\mathrm{MgO}$ ceramic and single crystal samples when excited at $265 \mathrm{~nm}$ in the UV-Vis (left) and NIR ranges (right). The inset in the right figure expands the spectral data around 700 $\mathrm{nm}$. 
1

2

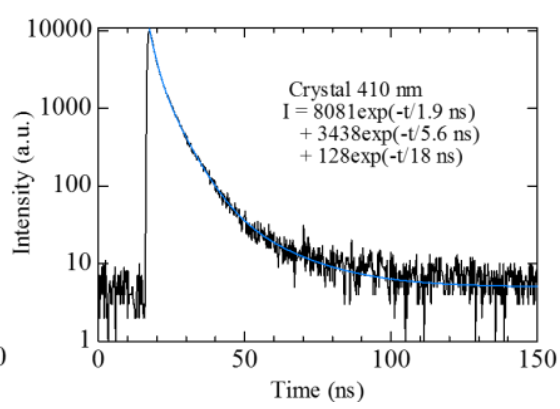

Fig. 5 PL decay time profiles of $\mathrm{MgO}$ ceramic (left) and single crystal (right) samples under $280 \mathrm{~nm}$ excitation while monitoring the $410 \mathrm{~nm}$ emission. 
1

2

3

4

5

7

8

9

10

11

12

13

14

15

16

17

18

19

20

21

22

23

24

25

26

27

28

29

30

31

32

33

34

35

36

37

38

39

40

41

42

43

44

45

46

47

48

49

50

51

52

53

54

55

56

57

58

59

60

61

62

63

64

65

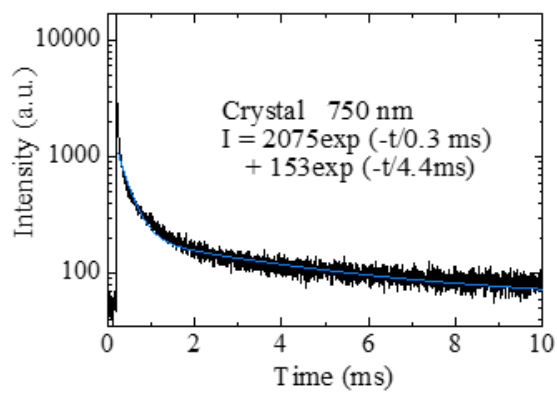

Fig. $6 \mathrm{PL}$ decay time profiles of $\mathrm{MgO}$ single crystal sample under $280 \mathrm{~nm}$ excitation while monitoring the $750 \mathrm{~nm}$ emission. 
1

2 3
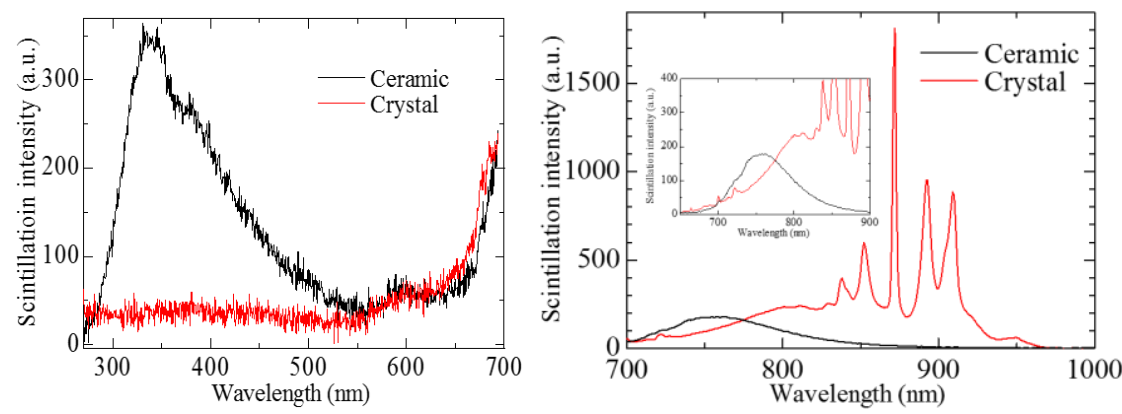

Fig. $7 \mathrm{X}$-ray induced scintillation spectra of the $\mathrm{MgO}$ ceramic and the single crystal samples. The inset expands $650-950 \mathrm{~nm}$ regions. 
1

2

3

4

5

6

7

8

9

10

11

12

13

14

15

16

17

18

19

20

21

22

23

24

25

26

27

28

29

30

31

32

33

34

35

36

37

38

39

40

41

42

43

44

45

46

47

48

49

50

51

52

53

54

55

56

57

58

59

60

61

62

63

64

65
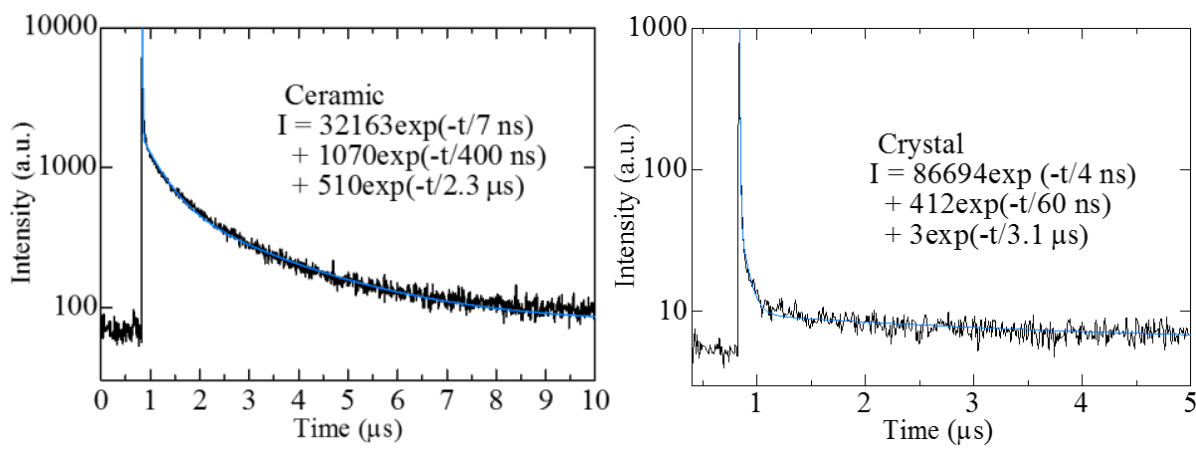

Fig. 8 Scintillation decay time profiles of the $\mathrm{MgO}$ ceramic (left) and single crystal (right) samples. 
1

2

3

4

5

6

7

8

9

10

11

12

13

14

15

16

17

18

19

20

21

22

23

24

25

26

27

28

29

30

31

32

33

34

35

36

37

38

39

40

41

42

43

44

45

46

47

48

49

50

51

52

53

54

55

56

57

58

59

60

61

62

63

64

65

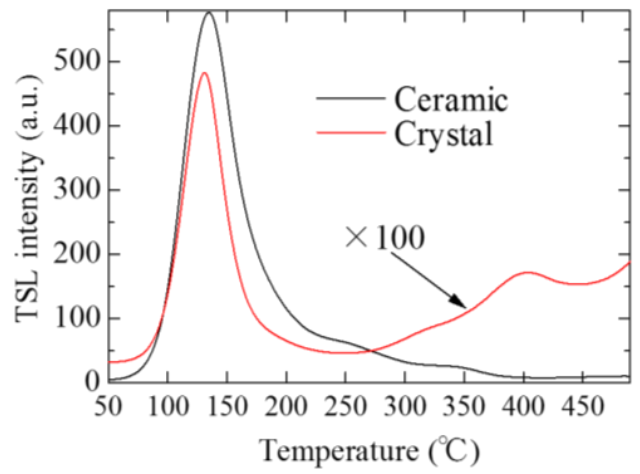

Fig. 9 TSL glow curve of the $\mathrm{MgO}$ ceramic and the single crystal after $1 \mathrm{~Gy} \mathrm{X}$-ray exposure. 
1

2

3

4

5

7

8

9

10

11

12

13

14

15

16

17

18

19

20

21

22

23

24

25

26

27

28

29

30

31

32

33

34

35

36

37

38

39

40

41

42

43

44

45

46

47

48

49

50

51

52

53

54

55

56

57

58

59

60

61

62

63

64

65

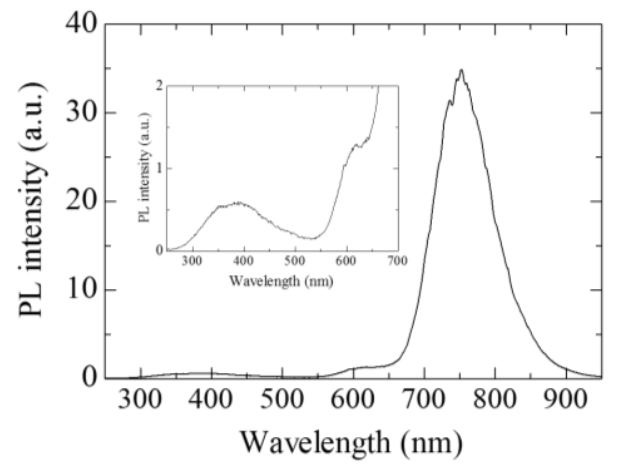

Fig. $10 \mathrm{TSL}$ spectra at $140^{\circ} \mathrm{C}$ glow peak of the $\mathrm{MgO}$ ceramic. The inset expands spectra from 250 to 700 $\mathrm{nm}$ 
1

2

3

4

5

6

7

8

9

10

11

12

13

14

15

16

17

18

19

20

21

22

23

24

25

26

27

28

29

30

31

32

33

34

35

36

37

38

39

40

41

42

43

44

45

46

47

48

49

50

51

52

53

54

55

56

57

58

59

60

61

62

63

64

65

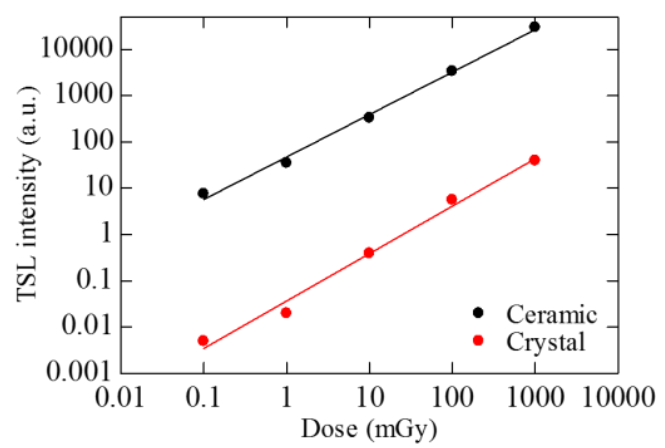

Fig. 11 Dose response curve of the MgO ceramic and the single crystal. The TSL signal used here is the peak intensity observed at $140^{\circ} \mathrm{C}$. 